\title{
EBV-Associated Gastric Carcinoma Arising in the Remnant Stomach: A Case Report and Review of the Literatures
}

\author{
Ran Hong* \\ Department of Pathology, College of Medicine, Chosun University, Korea
}

Submission: February 06, 2020; Published: February 18, 2020

*Corresponding author: Ran Hong, Department of Pathology, College of Medicine, Chosun University 309 Philmundaero, Dong-gu, Gwangju 61452, Korea

Abstract

Background: EBV-associated Gastric Carcinoma (EBVaGC) is defined by the presence of EBV in cancer cells, which is detected by EBVEncoded RNA (EBER) in situ hybridization. This entity is one of the four sub type of gastric cancer based on molecular biology according to recent proposal by The Cancer Genome Atlas (TCGA).

Case Presentation: Herein I report the case of a 72-year-old man with severe anemia. In the evaluation of the cause of anemia, malignant tumor was detected and underwent gastrectomy. The tumor infiltrated to the muscle proper and no lymph node metastasis was detected. The EBER in situ hybridization was positive, and prominent immune cells infiltration were identified. Pathological examination revealed it as EBV associated Gastric Carcinoma (EBVaGC).

Conclusion: This type of tumor is a specific entity of gastric carcinoma based on molecular abnormality with favorable prognosis compared with conventional adenocarcinoma. histological hallmark such as marked intra- or peritumoral immune cell infiltration and positivity in the EBER in situ hybridization will be helpful in the diagnosis.

Keywords: EBV, EBER in situ hybridization, lymphoepithelioma, adenocarcinoma

Abbrevations: EBVaGC: EBV-Associated Gastric Carcinoma; EBER: EBV-Encoded RNA; TCGA: The Cancer Genome Atlas; EBV: Epstein-Barr Virus; GCs: Gastric Carcinomas; CLR: Crohn's Disease-like Lymphphoid Reaction; CA: Conventional Adenocarcinoma; TCGA: The Cancer Genome Atlas Research Network; PD-L: Programmed Death Receptor-Ligand; EGD: Esophagogastroduodenoscopy; CT: Computed Tomography; LELC: Lymphoepithelioma-like Carcinoma; TILs: Tumor-Infiltrating Lymphocytes; GRC: Gastric Remnant Carcinoma; LN: Lymph Node; TMIT 1:

Tumor Microenvironment Immune Type 1; AI: Apoptosis Index

\section{Background}

Epstein-Barr virus (EBV) is a ubiquitous oncogenic r-type herpes virus discovered from a Burkitt lymphoma in 1964 [1,2]. Besides Burkitt lymphoma, it is comprehended in the etiology of several malignancies such as Hodgkin's lymphoma, nasal NK/T cell lymphoma, peripheral T-cell lymphoma, post-transplant lymphoproliferative disease, nasopharyngeal carcinoma, and a subset of gastric carcinomas (GCs) [2-4]. Histologically, EBV-associated gastric carcinoma (EBVaGC) is characterized by marked immune cell infiltration, and By Song et al, [5], which is classified into three histological subtype according to the cellular immune response affecting prognosis; lymphoepithelioma-like carcinoma (LELC), carcinoma with Crohn's disease-like lymphphoid reaction (CLR), and conventional adenocarcinoma (CA). EBVaGC is also one of definite subtypes of GCs based on molecular characterization classified by The Cancer Genome Atlas Research network (TCGA) (6): EBV-positive tumors, microsatellite-instable tumors, genomically stable tumors, and tumors with chromosomal instability. EBV-positive tumors display recurrent PIK3CA mutations, extreme DNA hypermethylation, and enhanced expression of JAK2, CD274 (also known as programmed death receptor-ligand 
[PD-L] 1), and PDCD1LG2 (also known as PD-L 2) providing a roadmap for patients stratification and trials of targeted therapies [6]. Herein we report a case of EBVaGC with mixed histologic features and review the literatures.

\section{Case Report}

A 72-year-old man with severe anemia underwent Esophagogastroduodenoscopy (EGD) to evaluation for the cause of severe anemia in the local clinic. He has a previous history of subtotal gastrectomy with Billoth II anastomosis by gastric ulcer perforation in 1997. The EGD resulted in the detection of large lesions showing irregular surface and central ulceration in the anastomosis site. The pathologic examination of specimen of endoscopic biopsy revealed it to be moderately differentiated adenocarcinoma. And, suspicious wall thickening in remnant stomach and anastomosis site with significant enlargement of the lymph nodes in lesser sac and LUQ abdomen was detected by a Computed Tomography (CT) of the abdomen. Therefore, total gastrectomy of residual stomach was performed. Grossly, a 13.0*7.0 cm-sized, C-shaped ulcerofungating lesion was found (Figure 1). Microscopically, there were 3 different histologic feature, well-formed glandular proliferation (Figure 2A), anastomosing island, nest, and cords (Figure 2B) and diffuse sheets of tumor cells (Figure 2C), which lesions have distinct boundaries. Tumor cells have large vesicular nuclei and prominent nucleoli and were admixed with plump inflammatory cells such as lymphocytes, plasma cells, histiocytes and occasional neutrophils or eosinophils. The tumor cells infiltrated to muscularis propria (pT2) and there was no lymph node metastasis or lymphovascular invasion. The examination of EBER (Ebstein Barr Virus-encoded RNA) in situ hybridization show strong positivity in all areas of tumor cells (Figure 2D).

Figure 1: Grossly, C-shaped ulcerofungating lesion is found.

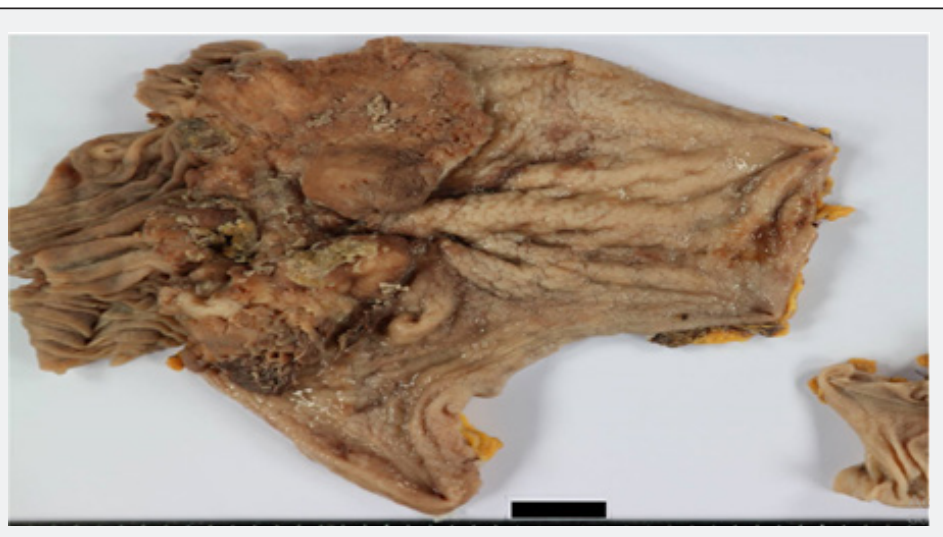

.

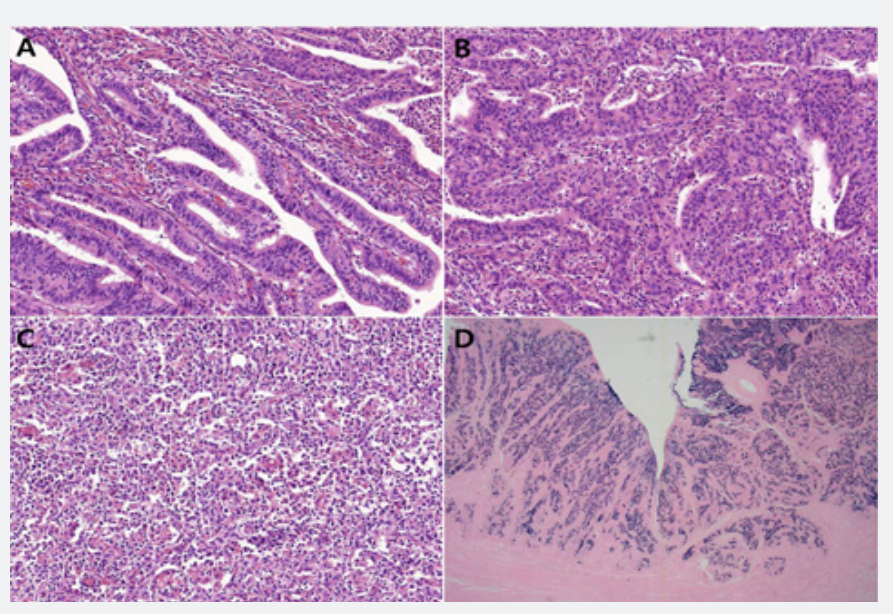

Figure 2: This tumor have various microscopic features, well-formed glandular proliferation A) anastomosing island, nest, and cords B) and diffuse sheets of tumor cells $\mathrm{C}$ ), which lesions have distinct boundaries. All of the tumor cells show positivity in EBER in situ hybridization (D).

Conclusively, I diagnosed the present case as EBV-associated gastric carcinoma (EBVaGC). Herein, I report a case of EBV-asso- ciated adenocarcinoma, which is a one of 4 distinct type of gastric cancer in the molecular base. 


\section{Discussion}

EBV-associated gastric carcinoma (EBVaGC) is defined by the presence of EBV in neoplastic cells, that is, the monoclonal proliferation of carcinoma cells with latent EBV infection, which is about $10 \%$ (range from $1.3 \%$ to $20.1 \%$ ) of all GC [7-9]. EBV infection can be demonstrated by in situ hybridization targeted at EBV-encoded small RNA (EBER). Cancer cells of EBVaGC contain EBV DNA sequences, and the EBV terminal repeat sequences have uniform lengths, implying that the tumor can arise from a single EBV-infected cell and that the EBV genome persists during malignant transformation and proliferation [10,11]. Chen et al, [12] noted that EBVaGC shows global and nonrandom CpG-island methylation of the promoter region of many cancer-related genes causing downregulation of their expression. And they proposed that the uniform presence of EBV-encoded small RNA in cancer cells but not in the normal cells, the detection of monoclonal EBV episomes in EBVaGC, and the elevation of IgG and IgA antibodies against viral capsid several month before the diagnosis of EBVaGC [13] suggests that EBV play an etiological role in gastric carcinogenesis.

In 2014, The Cancer Genome Atlas (TCGA) research network [6] proposed a new molecular classification of GCs into four subtypes: EBV-positive tumors, microsatellite-unstable tumors, genomically stable tumors, and tumors with chromosomal instability. EBV-positive tumors display recurrent PIK3CA mutations, extreme DNA hypermethylation, and amplification of JAK2, CD274 (known as PD-L1), and PDCD1LG2 (known as PD-L2). Setia et al, [14] identified five groups of GC in 2016; EBV (+) GC, microsatellite-instable, and GC with aberrant E-cadherin expression, GC with aberrant p53 expression, and GC with normal p53 expression. The EBVaGC showed a strong association with PD-L1. The EBV and microsatellite-instable related adenocarcinomas showed a trend for superior survival. This classification corresponds to the previous classification and offers a simplified and less expensive algorithm. Recent another classification of GCs by Ahn et al, [15] in 2017 on the basis of protein or mRNA expression of MLH-1, E-cadherin, p53, and EBV divided GCs into five subtypes which presented distinct clinicopathologic characteristics and corresponded to the previous classifications of GCs; EBV(+) tumors, aberrant MLH1 expression, aberrant E-cadherin expression, aberrant p53 expression and normal p53 expression. Of which, the EBV (+) tumors were mostly poorly differentiated type and characteristic molecular abnormalities such as mutation of PIK3CA, ARID1A, and BCOR, CDKN2A promoter hypermethylation, amplification of PDL1, PDL2, and JAK2. Like these, EBVaGC is a one of the distinct entities of the GCs.

Histologically, EBVaGC is characterized by marked intra- or peritumoral immune cell infiltration. Marked intra- or peritumoral immune cell infiltration is commonly observed in EBVaGC specimens. By Song et al, [5], they classified EBVaGC into three subtypes in the basis of the cellular immune responses affecting prognosis: 1) lymphoepithelioma-like carcinoma (LELC), 2) carcinoma with Crohn's disease-like lymphoid reaction (CLR), and 3) conventional adenocarcinoma (CA). Typical microscopic findings of LELC was like this; a well-defined margin, dense lymphocytic infiltration (the number of tumor-infiltrating lymphocytes (TILs) was greater than tumor cells), indistinct cytoplasmic borders and a syncytial growth pattern with poorly formed glandular structures, and no desmoplasia. CLR was characterized by patchy lymphocytic infiltration with three or more lymphoid follicles with active germinal centers, lower number of lymphocytes than tumor cells, frequent tubule or gland formation, and complete absence of minimal desmoplasia. In contrast to LELC and CLR, CAs show scattered lymphocytic with prominent desmoplasia in the absence of lymphoid follicles. Patients with LELC subtype of EBVaGC show the best overall and disease-free survival followed by CLR. So, this histologic subclassification of EBVaGC into 3 distinct subsets seems to be a powerful prognostic parameter. According to this histologic classification, the present case is correspond to the mixed EBVaGC with LELC, CLR, and CA, however, the portion of CA of the present case shows not only extensive intra- and peritumoral lymphocytic infiltration but also well-formed prominent glandular structure.

According to the study of the clinopathologic characteristics of EBVaGC in Japan by Yanagi et al, [16], various clinicopathologic factors of EBVaGC are significantly different from those of EBV negative GCs (EBVnGCs), that is, EBVaGC show male predominance, upper stomach, early stage (lower invasion depth), less lymphatic invasion, and dense lymphoid stroma. Chen et al, [12] also reported that EBVaGC show male predominance, predisposition to the proximal stomach, and a high proportion in diffusetype GCs. Interestingly, they reported that the frequency of EBVa$\mathrm{GC}$ in gastric remnant carcinoma (GRC) is significantly higher than that in conventional GC (CGC) which occurs in the intact stomach.

The recent meta-analysis of clinicopathological features of EBVaGC by Qiao et al, [17] identified that EBV infection rate was significantly higher in males and in diffuse cancer type, and no marked association with lymph node (LN) metastasis, age and pathologic tumor stage such as invasion depth. The predominance of male in the EBVaGC is interesting feature. So far, there have been no study showing female predominance. The male predominance suggest $s$ that lifestyle and occupational factors that are common among males [18] and sex-related hormonal and/or immune factors [12] may contribute to the development of EBVaGC. Anonther characteristic feature of EBVaGC is that incidence of EBVaGC in GRC is significantly higher than that in CGC occurring in the intact stomach [12]. GRC is defined as a GC occurring in the gastric stump at least 5 years after surgery for benign diseases such as gastric ulcer and duodenal ulcer $[19,20]$. The proportion of EBVaGC in GRC is $27 \%$ to $42 \%$, which was 3 to 4 times higher than that in CGC [21-24]. The present case is also a GC arising in the remnant stomach after subtotal gastrectomy due to ulcer perforation. 
Chen et al, [25] also investigated the EBV genome polymorphisms and clinicopathologic features of EBVaGC in GRC and CGC in Guangzhou, in which they identified that the EBV genome polymorphisms and clinicopathologic features of EBVaGC in GRC were similar to those in CGC, whereas the proportion of EBVaGC in GRC was significantly higher than that in CGC, which result suggest that the injuries of remnant gastric tissue and/or changes of the microenvironment may be involved in the development of EBVaGC. Kaizaki et al, [26] reported that in the remnant stomach after gastrectomy with Billroth2 reconstruction, performed for benign disease, long-term inflammation together with EBV infection, may have promoted the development of GC.

The most characteristic histologic finding of EBVaGC is a prominent cellular immune reaction. By Song et al, [5], especially in the subtype of LELC and CR of EBVaGC, the number of TILs increases. This histologic hallmark of EBVaGC may suggest that the interaction between tumor cells and tumor environment plays a critical role in the progression of EBVaGC. Several studies evaluated that the immune cells more common in EBVaGC are CD8+ cytotoxic T lymphocytes (CTLs) and mature dendritic cells compared with EBVnGC [27-30]. By Zhang et al, [31], In vitro, these (CTLs) EBVaGC could specifically kill autologous EBV-transformed lymphoblastoid cells, which immune responses may be associated with the favorable prognosis and low LN metastasis, low lymphovascular invasion and low invasion depth. In the study by Min et al, [31], especially, patients with LELC subtype of EBVaGC show the best prognosis followed by CLR and CA patients. Although the exact cellular characteristics or their roles are not certain at the present time, lymphocytic infiltration around tumor can be considered as a host immune reaction against tumor cells, and more dense lymphocytic infiltration could be considered as a stronger host immune reaction [5]. In the study about tumor microenvironment immune type in GC by Valentini et al, [32], a high rate of infiltration of CTLs is a characteristic of $\operatorname{EBV}(+)$ GC, Which immune reaction is belonged to tumor microenvironment immune type I (TMIT 1) out of classification based on the results of immunohistochemical expression of PD-L1 on TCs and CD8+ TILs density. According to this classification, the patients were categorized into the four following TMITs: I (PD-L1+/CD8+ high, adaptive immune resistance), II (PD-L1-/CD8+ low, immune ignorance type), III (PD-L1+/CD8+ low, intrinsic induction of PD L1) and IV (PD L1-/CD8+ high, tolerant tumors) [33].

The uncoordinated apoptosis and proliferation is the major cause for tumor development and progression. Fas/FasL is an important cell apoptosis signal pathway, also plays roles in immune surveillance. By He et al, [34], the expression of Fas and FasL in GC was significantly different from normal gastric tissues, which indicates that GC development is related to abnormal expression of Fas and FasL. Also, the apoptosis index (AI) and intensity of Fas expression in EBVaGC was significantly lower than in EBVnGC. They suggested that upregulation of FasL expression and de- creased apoptosis of TIL were favorable for tumor cells escape the immune surveillance and for tumor development and progression. Because of limited understanding of this newly defined entity, treatment of EBVaGC is the same as that of their counterpart, EBVnGC. That is, the treatment of GC just depend on the pTNM stage irrespective of EBV status. However, so far several treatment approaches specific to EBVaGC have been presented. One promising approach is presented by Jung et al, [35], they suggested the use of demethylating agents, such as 5-aza cytidine, to recover the lytic infection of EBV. Another possible therapeutic approach is by DX al [36] is to activate viral thymidine kinase by a proteosome inhibitor, such as bortezomib. The reactivation coupled with the administration of a radiolabeled substrate would theoretically result in specific targeting of the radioactive end-product to the EBVaGC. However, given the efficiency and the side effects of these agents, these approaches have not commercialized so far and should be studied further.

The precise role of EBV in carcinogenesis in stomach is not fully understood. Further studies are necessary to examine the interaction among EBV infection and other clinicopathologic factors such as environmental and genetic factors. Especially, considering the prognostic significances of host cellular immune reaction, further elucidation of the precise mechanisms of host cellular immune reaction may guide the development of specific therapeutic strategy to EBVaGC including immunotherapy.

\section{Authors' Contributions}

Ran Hong designed, wrote and revised the manuscript.

\section{Funding}

This study was supported by research funds from Chosun University Hospital, Republic of Korea, 2017.

\section{Ethics Approval and Consent to Participate}

The study was approved by the ethics committee of Chosun University Hospital (Institutional review Board of Chosun university hospital, Gwangju, Korea), who waived the requirement for written informed consent due to the nature of the study.

\section{Consent for Publication}

All data published here are under the consent for publication.

\section{References}

1. Zur Hausen H, Schulte-Holthausen H, Klein G, Henle W, Henle G, et al. (1970) EBV DNA in biopsies of Burkitt tumours and anaplastic carcinomas of the nasopharynx. Nature 228(5276): 1056-1058.

2. Young LS, Rickinson AB (2004) Epstein-Barr virus: 40 years on. Nat Rev Cancer 4(10): 757-768.

3. Epstein MA, Achong BG, Barr YM (1964) Virus particles in cultured lymphoblasts from burkitt's lymphoma. Lancet 1(7335): 702-703.

4. Epstein MA, Barr YM (1964) Cultivation in vitro of human lymphoblasts from burkitt's malignant lymphoma. Lancet 1(7327): 252-253. 
5. Song HJ, Kim KM (2011) Pathology of epstein-barr virus-associated gastric carcinoma and its relationship to prognosis. Gut Liver 5(2): 143-148.

6. (2014) The Cancer Genome Atlas Research network.

7. Akiba S, Koriyama C, Herrera-Goepfert R, Eizuru Y (2008) EpsteinBarr virus associated gastric carcinoma: epidemiological and clinicopathological features. Cancer Sci 99(2): 195-201.

8. Lee JH, Kim SH, Han SH, An JS, Lee ES, et al. (2009) Clinicopathological and molecular characteristics of Epstein-Barr virus-associated gastric carcinoma: a meta-analysis. J Gastroenterol Hepatol 24(3): 354-365.

9. Uozaki H, Fukayama M (2008) Epstein-Barr virus and gastric carcinoma-viral carcinogenesis through epigenetic mechanisms. Int Clin Exp Pathol 1(3): 198-216.

10. Fukayama M, Hayashi Y, Iwasaki Y, Chong J, Ooba T, et al. (1994) Epstein-Barr virus-associated gastric carcinoma and Epstein-Barr virus infection of the stomach. Lab Invest 71(1): 73-81.

11. Busson P, Keryer C, Ooka T, Corbex M (2004) EBV-associated nasopharyngeal carcinomas: from epidemiology to virus-targeting strategies. Trends Microbiol 12(8): 356-360.

12. Chen JN, He D, Tang F, Shao CK (2012) Epstein-Barr Virus-associated Gastric Carcinoma A Newly Defined Entity. J Clin Gastroenterol 46 (4): 262-271.

13. Levine PH, Stemmermann G, Lennette ET, Hildesheim A, Shibata D, et al. (1995) Elevated antibody titers to Epstein-Barr virus prior to the diagnosis of Epstein-Barr-virus-associated gastric adenocarcinoma. Int J Cancer 60(5): 642-644.

14. Setia N, Agoston AT, Han HS, Mullen JT, Duda DG, et al. (2016) A protein and mRNA expression-based classification of gastric cancer. Mod Pathol 29(7): 772-784.

15. Ahn S, Lee SJ, Kim Y, Kim A, Shin N, et al. (2017) High-throughput protein and mRNA expressionbased classification of gastric cancers can identify clinically distinct subtypes, concordant with recent molecular classifications. Am J Surg Pathol 41(1): 106-115.

16. Yanagi A, Nishikawa J, Shimokuri K, Shuto T, Takagi T, et al. (2019) Clinicopathologic Characteristics of Epstein-Barr Virus-Associated Gastric Cancer Over the Past Decade in Japan. Microorganisms 7(9): E305.

17. Qiao YW, Zhao XQ Liu J, Yang WJ (2019) Clinicopathological features of Epstein-Barr virus-associated gastric carcinoma: A systematic review and meta-analysis. JBUON 24(3): 1092-1099.

18. Koriyama C, Akiba S, Minakami Y, Eizuru Y (2005) Environmental factors related to Epstein-Barr virus-associated gastric cancer in Japan. J Exp Clin Cancer Res 24(4): 547-553.

19. Sinning C, Schaefer N, Standop J, Overhaus M, Hirner A, et al. (2007) Gastric stump carcinoma-epidemiology and current concepts in pathogenesis and treatment. Eur J Surg Oncol 33(2): 133-139.

20. Safatle-Ribeiro AV, Ribeiro U Jr, Reynolds JC (1998) Gastric stump cancer: what is the risk? Dig Dis 16(3): 159-168.

21. Yamamoto N, Tokunaga M, Uemura Y, Tanaka S, Shirahama H, et al. (1994) Epstein-Barr virus and gastric remnant cancer. Cancer 74(3) 805-809.

22. Chang MS, Lee JH, Kim JP, Kim HS, Lee HS, et al. (2000) Microsatellite instability and Epstein-Barr virus infection in gastric remnant cancers. Pathol Int 50(6): 486-492.
23. Baas IO, van Rees BP, Musler A, Craanen ME, Tytgat GN, et al. (1998) Helicobacter pylori and Epstein-Barr virus infection and the p53 tumour suppressor pathway in gastric stump cancer compared with carcinoma in the non-operated stomach. J Clin Pathol 51(9): 662-666.

24. Nishikawa J, Yanai H, Hirano A, Okamoto T, Nakamura H, et al. (2002) High prevalence of Epstein-Barr virus in gastric remnant carcinoma after BillrothII reconstruction. Scand J Gastroenterol 37(7): 825-829.

25. Chen JN, Jiang Y, Li HG, Ding YG, Fan XJ, et al. (2011) Epstein-Barr virus genome polymorphisms of Epstein-Barr virus-associated gastric carcinoma in gastric remnant carcinoma in Guangzhou, southern China, an endemic area of nasopharyngeal carcinoma. Virus Res 160(1-2): 191-199.

26. Kaizaki Y, Hosokawa O, Sakurai S, Fukayama M (2005) Epstein-Barr virus associated gastric carcinoma in the remnant stomach: de novo and metachronous gastric remnant carcinoma. J Gastroenterol 40(6): 570-577.

27. Cho J, Kang MS, Kim KM (2016) Epstein-Barr Virus-Associated Gastric Carcinoma and Specific Features of the Accompanying Immune Response. J Gastric Cancer16(1): 1-7.

28. Gulley ML, Pulitzer DR, Eagan PA, Schneider BG (1996) EpsteinBarr virus infection is an early event in gastric carcinogenesis and is independent of bcl-2 expression and p53 accumulation. Hum Pathol 27(1): 20-27.

29. Chang MS, Lee HS, Kim CW, Kim YI, Kim WH (2001) Clinicopathologic characteristics of Epstein-Barr virus-incorporated gastric cancers in Korea. Pathol Res Pract 197(6): 395-400.

30. Lee HS, Chang MS, Yang HK, Lee BL, Kim WH (2004) Epstein-barr viruspositive gastric carcinoma has a distinct protein expression profile in comparison with epstein-barr virus-negative carcinoma. Clin Cancer Res 10(5): 1698-1705.

31. Min BH, Tae CH, Ahn SM, Kang SY, Woo SY, et al. (2016) EpsteinBarr virus infection serves as an independent predictor of survival in patients with lymphoepithelioma-like gastric carcinoma Gastric Cancer 19(3): 852-859.

32. Valentini AM, Di Pinto F, Coletta S, Guerra V, Armentano R, et al. (2019) Tumor microenvironment immune types in gastric cancer are associated with mismatch repair however, not HER2 status. Oncol Lett 18(2): 1775-1785.

33. Taube JM, Anders RA, Young GD, Xu H, Sharma R, et al. (2012) Colocalization of inflammatory response with B7h1 expression in human melanocytic lesions supports an adaptive resistance mechanism of immune escape. Sci Transl Med 4(127): 127ra37.

34. He D, Xiao L, Chen JN, Liang Q, Shao CK (2010) Correlation of Fas/FasL expression to cell apoptosis in Epstein-Barr virus-associated gastric carcinoma (EBVaGC). Chinese J Cancer 29(3): 283-287.

35. Jung EJ, Lee YM, Lee BL, Cahng MS, Kim WH (2007) Lytic induction and apoptosis of Epstein-Barr virus-associated gastric cancer cell line with epigenetic modifiers and ganciclovir. Cancer Lett 247(1): 77-83.

36. Fu DX, Tanhehco Y, Chen J, Foss CA, Fox JJ, et al. (2008) Bortezomibinduced enzyme-targeted radiation therapy in herpesvirus-associated tumors. Nat Med 14(10): 1118-1122. 
CC Commons Attribution 4.0 License BY
Your next submission with JuniperPublishers will reach you the below assets

- Quality Editorial service

- Swift Peer Review

- Reprints availability

- E-prints Service

- Manuscript Podcast for convenient understanding

- Global attainment for your research

- Manuscript accessibility in different formats ( Pdf, E-pub, Full Text, audio)

- Unceasing customer service

Track the below URL for one-step submission https://juniperpublishers.com/online-submission.php 